\title{
Rezensionen
}

Erhorn, Jan, Schwier, Jürgen, Hampel, Petra (Hrsg.): Bewegung und Gesundheit in der Kita. Analysen und Konzepte für die Praxis. transcript Verlag, Bielefeld 2016, 248 Seiten, € 19,99 (D)

Im Zuge der Professionalisierung frühpädagogischer Fach-und Leitungskräfte sowie der qualitativen Weiterentwicklung pädagogischer Bildungsangebote fokussiert und verbindet dieser Sammelband die Bereiche Bewegung und Gesundheit als zentrale Themen für frühkindliche Bildungsprozesse. Dabei betonen die HerausgeberInnen die wichtige Rolle der Bewegung als Medium für die Entwicklung, das Lernen und die Gesundheitsbildung des Kindes. Die Beiträge konzentrieren sich auf Konzepte der Bewegungs- und Gesundheitsförderung, die zum einen Anregungen für einen bewegten Kita-Alltag bieten und zum anderen einen Beitrag zur Gesundheitsprävention der Mitarbeiterlnnen leisten sollen.

Schwier bietet in einem einführenden Kapitel einen Überblick über den vielschichtigen Gesundheitsbegriff und stellt Bewegung als eine wesentliche Gesundheitsressource in der frühen Kindheit dar. Im folgenden Beitrag nimmt P. Hampel die psychische Gesundheit gezielter in den Blick und thematisiert Fördermöglichkeiten in der Kita. Auf Grundlage von Befunden zur gesundheitlichen Lage von Erzieherlnnen erweitern die folgenden beiden Kapitel die Zielgruppe der Gesundheitsbildung: Wagner skizziert verschiedene Ansätze einer verhaltens- und verhältnisorientierten Gesundheitsförderung in der Kita, während Schwier/Schwier ein bewegungsorientiertes Anti-StressTraining für pädagogische Fachkräfte vorstellen. Im nachfolgenden Abschnitt fokussiert J. Schwier die Merkmale und Formen des Spiels und deren Bedeutung für frühkindliche Entwicklungsprozesse. In einem gemeinsamen Exkurs mit Erhorn vertieft er im sechsten Kapitel die vorangehenden Ausführungen mittels der Analyse einer konkreten Spielsituation.

Die nachfolgenden Beiträge stellen die Auseinandersetzung mit dem Bewegungsbegriff in den Vordergrund. Der Darstellung des dialogischen Bewegungskonzepts und seiner Relevanz für die Bewegungserziehung und -förderung (Schwier) folgt im achten Kapitel eine Bestimmung der entwicklungsfördernden Potenziale der Bewegung und entsprechender Raumbedingungen (Erhorn). Ferner werden typische Aktionsformen der Kinder beschrieben, die von Erhorn im anschließenden Beitrag zur Inszenierung von Bewegung in der frühen Kindheit berücksichtigt werden. Der Autor gibt einen Überblick, wie Bewegungsangebote geplant, durchgeführt und ausgewertet werden können, und bietet als Gegenstand des zehnten Kapitels eine Übersicht über verschiedene Handlungsfelder, im direkten Kitaumfeld und in außerinstitutionellen Bewegungsräumen, zum direkten Praxistransfer. Schließlich werden in den Kapiteln elf und zwölf die Poten-

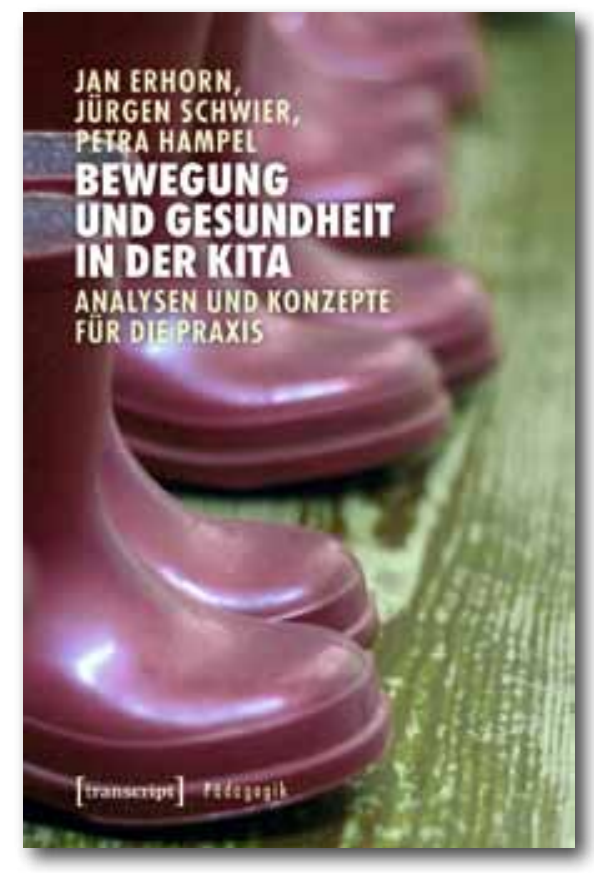

ziale bewegungsorientierter Arbeit im Kontext der aktuellen Themen Sprachförderung (Erhorn) sowie Inklusion und Partizipation (Schwier/Schwier) beleuchtet. Das Werk schließt mit einem Aufgabenteil $a b$, der als Grundlage für die Verwendung in der Lehre dienlich ist.

Die Autorlnnen dieses Buches geben einen umfassenden praxisnahen und wissenschaftlich fundierten Überblick über Konzepte und Ansätze der bewegungs- und spielorientierten Gesundheitsbildung und Entwicklungsförderung in der frühen Kindheit. Die Beiträge bauen inhaltlich aufeinander auf, bilden jedoch in sich abgeschlossene thematische Schwerpunkte, die auch 
unabhängig von den Folgebeiträgen gelesen und verstanden werden. Kompetenzorientiert bietet das Werk theoretische Wissensvermittlung auf der einen Seite, zeitgleich aber auch Strukturen zur Entwicklung von Fertigkeiten und personalen Kompetenzen auf der anderen Seite. Durchgehend formulierte
Fragen regen zur Reflexion des eigenen Handelns an und befähigen den Leser zur Analyse und Organisation der individuellen institutionellen Rahmenbedingungen.

Das Buch bietet einen wertvollen Beitrag zur Professionalisierung frühpädagogischer Fach- und Leitungs- kräfte im Anwendungsbereiche Bewegung und ermöglicht eine Verankerung des vielschichtigen Bewegungsverständnisses in der frühpädagogischen Aus- und Weiterbildungslandschaft.

Aida Kopic

\section{Kurz vorgestellt}

Monika Brunner, Christiane Müller, Silke Schwarz: Sprachspiele und Bewegungslieder für Kinder von 4 bis 8 Jahren. Schulz-Kirchner, Idstein, 2., überarbeitete und erweiterte Auflage 2016, 208 Seiten, $€$ 32,50 (D)

Ausgehend vom Heidelberger Vorschulscreening zur auditiv-kinästhetischen Wahrnehmung und Sprachverarbeitung (Brunner et al. 2001) entwerfen die Autorinnen eine Vielfalt von Sprachspielen und Übungsformen, die der Wahrnehmungsförderung dienen und den Schriftspracherwerb vorbereiten.

In einer kurzen Einführung werden die Merkmale auditiver Wahrnehmungsstörungen erläutert und ihre Auswirkungen auf die Lese- und Rechtschreibentwicklung beschrieben. Ergänzend werden die Bezüge zum Heidelberger Vorschulscreening dargestellt und die Möglichkeiten, daraus Förderbedarf und konkrete Förderziele abzuleiten.

Das Buch enthält Grundinformationen und Spielvorschläge für folgende Förder- und Therapieschwerpunkte:
- Lautanalyse

- Silbensegmentierung

- Phonematische Differenzierung

- Artikulomotorik

- Erkennen von Wortfamilien

- Erkennen von Reimwörtern

- Auditive Merkspanne

$\mathrm{Zu}$ all diesen Übungsbereichen werden vielfältige Spielmaterialien, Arbeitsblätter und Wortlisten bereitgestellt. Die Übungen lassen sich sowohl in (Klein-)Gruppen als auch in der Einzeltherapie nutzen und können von Erzieherinnen / Erziehern und Therapeutinnen/Therapeuten gleichermaßen sinnvoll angewandt werden.

\section{Verlagstext}

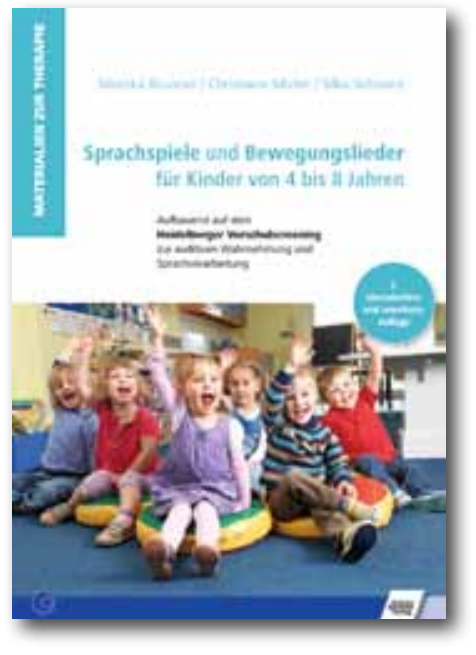

mot ${ }^{\text {rik }}$ 2 | 2017 